\title{
Germanistikstudium in Marokko - Probleme und Perspektiven
}

\author{
Abdelaziz Bouchara \\ Universität Casablanca, Germanistisches Seminar, Lotissement SMIRALDA Nr. 129, Lissasfa, Casablanca, \\ Marokko \\ E-mail: hanaae2001@yahoo.fr
}

\begin{abstract}
In many African countries, German Studies has recently come under increasing pressure. In view of the globalization of economic and cultural processes, as well as the wider acceptance of French and English, German Studies finds itself in growing competition. This is also true in Morocco. In this context, as elsewhere, it has become essential to seek alternatives and to become innovative. Thus the traditional $\mathrm{DaF}$ (German as a foreign language) also has to redefine its roll. In this paper the author presents a professionally oriented course consisting of three modules: (1) business German, (2) German for tourism, and (3) intercultural communication. In addition, the general conditions under which such modules are to be introduced will be discussed.
\end{abstract}

Keywords: German language and literature studies, German for business, German for tourism, intercultural communication

Schlüsselbegriffe: Germanistikstudium, Wirtschaftsdeutsch, Deutsch für den Tourismus, interkulturelle Kommunikation

\section{Einleitung}

Ich möchte an dieser Stelle aus einer Retrospektive heraus die aktuelle Situation der Germanistik und des Deutschen als Fremdsprache in Marokko skizzieren. Das Fach Deutsch steht ohne Zweifel unter Druck. Die Konkurrenz durch andere Sprachen, vornehmlich Französisch und das wachsende Interesse an Englisch, ist deutlich spürbar. Darüber hinaus nehmen bedauerlicherweise auch nach der sog. Universitätsreform die Inhalte und Zielsetzungen des Germanistikstudiums in keiner Weise Bezug auf Berufsfelder für Germanistik-Absolventen. Studierende der Germanistik sehen sich deshalb unter einem Legitimationsdruck, der den Nutzen des Wissenserwerbs an den späteren Karrierenchancen misst und das ausgerechnet in einer Zeit wachsender Arbeitslosigkeit, begleitet von Reizüberflutung und von zunehmender Orientierungslosigkeit in Wertefragen, die gerade auch bei vielen Jugendlichen zu Verweigerung und Abwehr führen. Wenn man dieser Einschätzung Glauben schenkt, dann scheinen wir es schon mit einer ernsthaften Krise zu tun zu haben, für die Marokko gewiss nur als Beispiel steht. Krisen beinhalten also die Chance, Altes zu überdenken, Auswege zu suchen und innovativ zu werden. Ich möchte in meinem Beitrag auf diese Punkte eingehen und zugleich Verbesserungsvorschläge anbieten. 


\section{Studienplan vor der Reform: ein kurzer Überblick}

Das Germanistikstudium ist als voller Studiengang durch drei Abteilungen an den Universitäten (Casablanca, Rabat und Fes) vertreten. Das vierjährige Studium (gültig bis zur Reform 2003) teilte sich in zwei Phasen, nämlich in das Grundstudium und das Hauptstudium, die jeweils zwei Jahre dauerten. Das Studienjahr beginnt im Oktober und endet mit einer Jahresendprüfung, die aus einem schriftlichen und einem mündlichen Teil besteht und im Juni durchgefürt wird. Wenn man die Prüfung nicht besteht, muss man das ganze Jahr wiederholen.

Im Grundstudium werden Grundkenntnisse der deutschen Sprache vermittelt und die vier Fertigkeiten (Hören, Sprechen, Lesen und Schreiben) trainiert, während im Hauptstudium die Sprachkenntnisse erweitert und vertieft werden. Die vier Fertigkeiten werden weiter gefördert und Übersetzungsfähigkeiten entwickelt sowie deutsche Geschichte und Landeskunde vermittelt. Hinzu kommen sprachwissenschaftliche und literarische Veranstaltungen. Am Ende des Studiums muss jeder Student eine Abschlussarbeit in deutscher Sprache anfertigen. Nach dem Studienabschluss mit der Licence können die Studierenden eine einjährige Referendarausbildung an der Ecole Normale Supérieure (ENS) machen, um die Lehrbefähigung für Deutsch als Fremdsprache an den Gymnasien zu bekommen. Die Licence ist ebenfalls Voraussetzung für eine Bewerbung am König-Fahd-Institut für Translation in Tanger (Ecole Supérieur Roi Fahd de Traduction - ESRFT), wo ein Übersetzerstudiengang für Arabisch/Deutsch/Französisch aufgebaut wurde.

Diese traditionelle Disziplinverteilung in Linguistik, Literaturwissenschaft und Landeskunde war m.E. so trocken erstellt, dass es die Denkfähigkeit nicht genug anregen konnte. In fast allen geisteswissenschaftlichen Fächern ist Frontalunterricht die vorherrschende Lehrform. Der Stoff wird vom Lehrer vorgetragen und in der Regel ohne eigene Reflexion von dem Studierenden memorisiert, damit er dann bei der Prüfung abrufbereit ist. Oft wird der Stoff auswendig gelernt, um dann gleich nach der Prüfung wieder vergessen zu werden. Deshalb konnten Germanisten in vielen Berufsfeldern nicht eingesetzt werden, denn das würde eine Professionalisierung des Studiengangs und eine Spezialisierung sowie eine kommunikative und interkulturelle Kompetenz voraussetzen (Übersetzen/Dolmetschen, interkulturelle Kommunikation, Fachsprachen z.B. Wirtschaftsdeutsch/Deutsch für den Beruf, Tourismus u.a.).

\section{Zur Frage der Curricula nach der Reform}

Gesellschaftliche Veränderungen und die schlechte Lage der Bildung in Marokko machten es dringend erforderlich, den Fremdsprachenunterricht den tatsächlichen Bedürfnissen anzupassen. Daher hat die Regierung in den Jahren 1990 bis 2000 mit Nachdruck daran gearbeitet, eine umfangreiche Bildungsreform durchzuführen. Für den Erwerb der deutschen Sprache und die Beschäftigung mit der deutschen Kultur sind dabei folgende Elemente wichtig:

- die wachsende Zusammenarbeit mit deutschen Firmen;

- der neue Boom des Tourismus;

- viele Studierende würden gerne zum Studium nach Deutschland gehen, auch nach der Einführung der Studiengebühren. 
Die im Jahr 2003 erfolgte Reform brachte die Studiendauer auf 3 Jahre bzw. 6 Semester. Am Ende jedes Semesters müssen die Endprüfungen für die ausgewählten Einheiten abgelegt werden. Die Teilnahme an den Kursen und die Zwischentests spielen bei der Leistungsbeurteilung ebenfalls eine große Rolle. Wenn ein Studierender bei einer Prüfung keinen Erfolg hat, muss er das ganze Modul im nächsten oder übernächsten Semester wiederholen.

Die gegenwärtige Universitätsrefom in Marokko bietet eine Chance zu Veränderungen, denn die Modularisierung der Studiengänge ermöglicht theoretisch zum einen eine individuelle Spezialisierung und zum anderen eine interdisziplinäre Kooperation und Ausbildung. Das aktuelle Studiensystem bietet den Studierenden bedauerlicherweise keine Möglichkeit, weitere Fächer innerhalb derselben Fakultät oder Universität mit dem Hauptfach zu kombinieren, wodurch ihre Berufschancen künftig erhöht werden könnten. Die inhaltliche Analyse zeigt außerdem, dass die Module nicht dafür geeignet sind, die Germanistikabsolventen zu Kompetenz und Handlungsfähigkeit z.B. in Wirtschaft, Tourismus, Übersetzen/Dolmetschen und interkultureller Kommunikation auszubilden. Es hat sich eigentlich nichts geändert, auch wenn der Inhalt des alten Systems in Form von Modulen in einen neuen Gesamtrahmen eingebettet ist. Ausgehend von dem DaF-Lehrplan in der jetzigen Version stellen wir fest, dass eine traditionelle Verteilung in Linguistik, Literaturwissenschaft und v.a. Landeskunde, wie vor der Reform, den Berufsperspektiven bei den Studierenden der Germanistik nicht entgegenkommt.

Als besonderes Defizit kann u.a. das Fehlen jeglicher Motivation angeführt werden. Ungewisse Zukunftsaussichten, auch angesichts von mangelhaften Studieninhalten, tragen nicht gerade dazu bei, die Studierenden stärker zu motivieren. Sie fördern im Gegenteil Unlust und ein unkontinuierliches Studium. Dies wirkt sich wiederum sehr negativ auf Deutsch als Fremdsprache in der Schule und im Gymnasium aus.

\section{Deutsch in der Schule und an der Universität: Eine gegenseitige Beeinflussung}

In Marokko beginnen die meisten Schulen mit Französisch als erster Fremdsprache. Im Gymnasium wählen die Schüler eine zweite Fremdsprache; bei dieser Fremdsprache handelt es sich in der Regel um Englisch. An einigen Gymnasien werden die Schüler dazu gezwungen, Deutsch oder Spanisch zu lernen. Sehr viele Schüler - obwohl es Ausnahmen gibt - mögen Deutsch nicht; sie lernen es nicht gern und erwarten schlechte Noten. Hauptursachen scheinen das grammatische Image, der Ruf ein schwierigen Sprache zu sein, das Ausbleiben unmittelbarer Erfolge im Anfangsunterricht und ein fehlendes Nützlichkeitsgefühl zu sein. Der letzte Faktor hängt mit der Stellung des Deutschen in Marokko und mit dessen praktischen Verwertbarkeit, die sehr beschränkt ist, zusammen. Dies ist den Eltern sehr bewusst und deshalb versuchen sie ihre Kinder bei der Wahl des Englischen als zweite Fremdsprache mit allen Mitteln zu unterstützen und sogar, wenn es nötig ist, das Gymnasium zu wechseln.

Aus diesen Bemerkungen wird klar, dass Deutsch als zweite Fremdsprache an marokkanischen Gymnasien von Streichungen bedroht ist. An einigen Gymnasien in Casablanca wurde Deutsch abgeschafft. Die Deutschlehrer werden dann woanders hin verlegt oder der Direktor sucht eine Beschäftigung für sie in der Bibliothek des Gymnasiums. Die Einführung von Deutsch als zweiter Fremdsprache in die Sekundarschule (Collège) - ein Jahr 
vor dem Gymnasium - mit dem gleichen Status wie andere Fremdsprachen wird deshalb als offizielle Lösung, auch seitens des Goethe-Instituts, angesehen. Diese Entwicklung könnte ganz allgemein zu einer stärkeren Position für das Fach Deutsch führen, wenn die Kontinuität dieses Schrittes im Gymnasium gewährleistet wäre. Das Gegenteil ist leider der Fall: Sobald die Schüler im Gymnasium sind, stellt man fest, dass sie - aufgrund der oben genannten Gründe - Deutsch als Fremdsprache nicht weiterführen möchten. In diesem Fall wird meistens Englisch gewählt, denn Deutsch als Fremdsprache wird merkwürdigerweise gar nicht angeboten oder ist schon abgeschafft. Diese schwierige Lage des Deutschen beeinflusst nicht nur die Einstellung der Deutschlerner, sondern auch die integrative Motivation, die bestimmend für den Unterrichtserfolg sein kann.

Die solchermaßen beschriebene Stellung der deutschen Sprache im Rahmen des schulischen Fremdsprachenunterrichts wirft Licht auf das Profil der Studierenden der Germanistik zu Beginn des Studiums. Das Studienfach Germanistik wird in Marokko in den wenigsten Fällen aus Neigung und Interesse gewählt. Es gilt: Lieber einen Platz für ein Germanistikstudium als gar keinen Studienplatz. Die Germanistik rangiert deshalb auf der Beliebtheitsskala auf einem der letzten Plätze. Wenn man als Hochschullehrer die Entwicklung der Einschreibungen für die Germanistik vergleicht, stellt man fest, dass der Anteil der Studierenden, die das Studienfach Germanistik wählen, tatsächlich in höchst beunruhigender Weise zurückgegangen ist.

Wir befinden uns in einer dramatischen Spirale: Einerseits haben wir die Universität, andererseits die Sekundarschule und die Oberschule (Gymnasium). Die negativen Berufsperspektiven der Germanistikstudenten erzeugen bei den Deutschlernern in der Schule ein Desinteresse und Mangel an Motivation, was nach dem Abitur Unlust bei den Germanistikanfängern fördert oder $\mathrm{zu}$ einem unkontinuierlichen Studium führt. Diese gegenseitige Beeinflussung sollte man bei jeder Reformdiskussion zur Kenntnis nehmen. Daher ist die Regierung derzeit mit Nachdruck dabei, tiefgreifende Veränderungen innerhalb der Bildungsreform durchzuführen. Aber wie kann die Attraktivität des Studiengangs Germanistik verbessert werden?

In vielen Berufsfeldern konnten Germanistik-Absolventen eingesetzt werden, aber das setzt, wie schon erwähnt, eine Professionalisierung des Studiengangs und eine Spezialisierung voraus. Über diese Bedingung müssen sich die Germanisten vor Ort ernsthafte Gedanken machen. Das bedeutet, dass wir in Zukunft noch mehr die Bedürfnisse der Studierenden zu berücksichtigen haben, ebenso ist es notwendig sich zu überlegen, wie, wann, wo und in welchem Masse sprachliche Kompetenzen erhöht werden können, damit sowohl die Germanisten als auch die Deutschlehrer in ihrem späteren Leben effektiver mit der deutschen Sprache umgehen können. Ich plädiere daher dafür, den verstärkten Kontakt zur Praxis zu suchen.

\section{Wie sieht die Praxis aus, auf die die Studenten vorbereitet werden sollen?}

\subsection{Wirtschaftsdeutsch}

Im Rahmen der Umstrukturierungsdiskussion werden gegenwärtig neue Kurskonzepte diskutiert. Es wird u.a. überlegt, ob z.B. Wirtschaftsdeutschkurse angeboten werden sollen, die sowohl von Studierenden des Faches Deutsch als auch von Studierenden des Faches Wirtschaft besucht werden können und gleichzeitig für Geschäftsleute offen wären. Betonen 
möchte ich an dieser Stelle, dass die Relevanz der deutschen Sprache im Wirtschaftsbereich von vielen Studierenden und auch von Lehrkräften zu hoch eingeschätzt wird. Englisch ist und bleibt die wichtigste Wirtschaftssprache der Welt. Deutsche Fachsprachkenntnisse können allenfalls eine Zusatzqualifikation sein. Dennoch darf nicht übersehen werden, dass wegen der zunehmenden Globalisierung die Mehrsprachigkeit im Zentrum der Diskussion über Sprachen in Europa steht, wobei die deutsche Sprache als eine der wichtigsten Arbeitssprachen neben Englisch und Französisch, in der Europäischen Union eine wichtige Rolle spielt. Darüber hinaus wirkt sich die Beherrschung von Fremdsprachen auf den Handel mit anderssprachigen Partnern positiv aus. Wer die Sprache des Gegenübers sprechen kann, punktet aus psychologischer Sicht durch die unmittelbare Verständigung mit seinem Gesprächspartnern (vgl. Zhoo 2000:2-3) ${ }^{1}$. Die Euro-mediterrane Partnerschaft führt ebenfalls zu wachsenden Kontakten mit Deutschland und trägt dazu bei, dass die deutsche Sprache auf absehbare Zeit an Bedeutung gewinnen wird (vgl. Wilsch 2003:200-201). In diesem Zusammenhang sollte man auch die Tatsache berücksichtigen, dass es in Marokko eine große Anzahl von deutschen Firmenniederlassungen und marokkanischen Firmen mit DeutschlandKontakten gibt. Diese benötigen Arbeitskräfte, die zielgerichtet ausgebildet werden, und dazu gehören m. E. Kenntnisse in Wirtschaftsdeutsch. Auch viele Unternehmensvertreter, die nur Französisch und Englisch können, betonen, wie wichtig die deutsche Sprache im internationalen Geschäftverkehr sei. Bis vor kurzem wurden noch diejenigen bevorzugt, die neben Französisch Englischkenntnisse vorweisen konnten. Von daher sollte die Germanistik auch Lehrveranstaltungen aus dem Bereich Fachsprache, z.B. Wirtschaftdeutsch, anbieten, sodass unsere Studierenden des Faches Germanistik von den beruflichen Chancen profitieren können, die deutsch-marokkanische Industriebetriebe bieten. Eine entsprechende Ausbildung $\mathrm{zu}$ erhalten bedeutet, Elemente der Wirtschaft in das Germanistikstudium selbst zu integrieren. Dazu gehört, dass in den bereits üblichen Übersetzungsseminaren literarische durch Sachtexte (z.B. Texte wirtschaftlicher Natur) zu ersetzen sind. Dadurch erwerben die Studierenden für ihren künftigen Beruf eine höhere mündliche und schriftliche Kompetenz in der deutschen Sprache und verschaffen sich Vorteile im beruflichen Auftreten. Insbesondere erwerben sie Fähigkeiten in folgenden Bereichen:

- Handelskorrespondenz und Vorstellungsgespräche;

- Auftragsabwicklung (von der Anfrage bis zur Zahlung);

- Lesen von Wirtschaftstexten;

- Firmenpräsentation;

- Grafikkommentierung;

- Telefonkommunikation;

- Führen von Mitarbeiter-, Kunden- und Verkaufsgesprächen;

- Wirtschaftsleben in Deutschland mit den folgenden Kernbereichen: Wirtschaftsysteme, Sozialversicherung, Wirtschaftsindikatoren, Marketing, Bankenund Börsenwesen, Währungssysteme, Wirtschaftsgeographie, Europäische Union, Unternehmensformen usw.;

- Professionelle Übersetzung (Wirtschaft und Handel);

- Praxis im Rahmen eines Praktikums und eine praxisbezogene Projektarbeit.

\subsection{Deutsch für den Tourismus}

Das Germanistikstudium sollte auch dazu beitragen, eine Tätigkeit im prosperierenden Tourismusbereich $\mathrm{zu}$ finden, indem eine entsprechende Ausbildung für das Berufsfeld Tourismus entwickelt wird. Im Tourismus bieten sich nationale und internationale Aufstiegschancen. Beschäftigungsmöglichkeiten bestehen bei Airlines, 
Flughafenverwaltungen und Studienreisen sowie in allen Bereichen des Hotel- und Gastgewerbes (Hotels, Gaststätten, Pensionen, Restaurants usw.). Im Hinblick auf die Bedeutung Marokkos als Tourismusland sind qualifizierte Fachkräfte im Hotel- und Gastgewerbe laufend gefragt. Es gibt zwar gute Absolventen von Hotelfachschulen in Marokko, aber ihre sprachliche Ausbildung ist unzureichend. Die erworbenen Sprachkenntnisse von Germanistikabsolventen sind relativ gut, aber ihen fehlt die entsprechende fachliche Ausbildung. Das Angebot von Tourismusmodulen an der Universität oder eine intensive Sprachausbildung an den Tourismusinstituten könnte zu positiven Änderungen führen. In dem stark praxisorientierten Ausbildungsgang „Deutsch für den Tourismus" soll den Studierenden folgende Kenntnisse vermittelt werden:

- Eine fachbezogene Sprachausbildung (mündliche und schriftliche Kommunikation in der Hotel- und Tourismusbranche: allgemeine Gesprächstechnik, Telefongespräche und Dialoge; das Schreiben bzw. Bearbeiten von Geschäftsbriefen, Formulieren von Aufträgen, Reservierungen und Reklamationen);

- Betriebswirtschaft und Rechnungswesen (Konjunktur, Wirtschaftwachstum, Grundlagen des Rechnungswesens z.B. Tabellenkalkulation/Excel);

- Geographie des Tourismus und Tourismuspolitik;

- Tourismusmarketing (Marketingphilosophie und Konzepte, Marketingziele und Strategien);

- Computer-Reservierungssysteme und Branchensoftware;

- Praktikum und praxisbezogene Projektarbeit.

\subsection{Interkulturelle Kommunikation}

Die Praxis verlangt auch interkulturelle Kommunikationskompetenz, die in der heutigen globalisierten Welt ein Schlüsselfaktor in der internationalen Kommunikation ist. Wer interkulturell kompetent handeln will, muss sowohl die fremde wie die eigene Kultur verstehen. Insbesondere auf dem Weltwirtschaftsmarkt, der durch Konkurrenz, Leistungsdruck und internationale Verflechtungen gekennzeichnet ist, gewinnt interkulturelle Kommunikation immer mehr an Bedeutung. An dieser Stelle wird schon klar, dass das Thema interkulturelle Wirtschaftkommunikation nicht nur wissenschaftlich, sondern auch wirtschaftlich sehr interessant ist. Peinliche Missverständnisse zwischen Angehörigen verschiedener Kulturen führen immer wieder dazu, dass Aufträge verloren gehen und politische Verhandlungen scheitern. Sprachliche Kenntnisse reichen also nicht aus, um auf fremden Märkten Erfolg zu haben. Die Fähigkeit, sich in anderen Kulturen sicher zu bewegen, muss hinzukommen. In interkultureller Kommunikation sind die dazu erforderlichen Kenntnisse zu vermitteln. Es soll u.a. gezeigt werden, warum insbesondere (wirtschaftliche) Kommunikation zwischen Europäern und Arabern/Asiaten aufgrund verbaler und nonverbaler Kulturunterschiede schwierig ist. Sprach- und Kulturkenntnisse (soft skills) werden z.B. für den Umgang mit ausländischen Kunden gebraucht. Wer im Ausland ein Produkt verkaufen will, muss es dem Gesprächspartner möglichst leicht machen, das eigene Waren- und Dienstleistungsangebot zu verstehen und zu bewerten.

Das Curriculum DaF an der marokkanischen Universität sieht für die Studienjahre 1-3 eine Reihe von Lehrinhalten vor, die grob unter dem Begriff „Landeskunde“ subsumiert werden können. Diese Lehrinhalte haben zum Ziel, den Studirenden historisch-politische Themen vertraut $\mathrm{zu}$ machen und sind nicht dafür geeignet, die Germanistikabsolventen zur interkulturellen Kompetenz in interkulturellen Begegnungen auszubilden. Es gibt zwar Module, die die Bezeichnung ,interkulturelle Studien/études interculturelles“ tragen, aber die 
inhaltliche Analyse zeigt, dass es sich bei solchen Veranstaltungen eher um landeskundliche Themen handelt.

Die Studierenden sollen dagegen über das theoretische und methodische Wissen verfügen, mit dem sie den kulturellen Kontext, in dem die eigenen Handlungen und die Handlungen anderer stehen, erschließen können. Dabei sollen folgende Lernziele beachtet werden, damit die Studierenden befähigt werden:

- die für die interkulturelle Kommunikation wichtigen Theorien und Methoden aus der Kulturanthropologie, der kulturvergleichenden Psychologie, der Pragmatischen Linguistik, der Wirtschaftswissenschaft und der Pädagogik zu verstehen und anzuwenden,

- die Ursachen von Problemen und Konflikten, die sich in interkulturellen Situationen ergeben können, realistisch einzuschätzen und geeignete Lösungsmöglichkeiten zu finden.

Künftige Berufsfelder der Absolventen könnten international agierende Kultur-, Wissenschafts- und Bildungsinstitutionen bzw. politische Einrichtungen und Wirtschaftsunternehmen im Rahmen der deutsch-marokkanischen Zusammenarbeit sein. ${ }^{2}$

\section{Perspektivenwechsel}

Es gilt das Niveau des Germanistikstudiums zu verbessern, um Wettbewerbsfähigkeit zu erreichen. In vielen Ländern, z.B. Thailand, China und Taiwan, dienen die modernen westlichen Sprachen Englisch, Französisch und Deutsch als „Werkzeug“, mit dem der Anschluss an das westliche Niveau der technologischen Entwicklung erleichtert werden soll. Die Marokkaner sollten ebenfalls dieser Zielbestimmung des Unterrichts in der deutschmarokkanischen Zusammenarbeit folgen und bei der Erstellung der Curricula berücksichtigen. Die neue Germanistik in Marokko ist immer noch im sog. Dreiermodell (Literaturwissenschaft, Linguistik und Landeskunde) organisiert und bietet gegenwärtig nur noch eine allgemeine Ausbildung ohne Spezialisierungsmöglichkeiten. Deshalb ist eine offene Diskussion bei den Germanisten in Marokko nötig, um zu verhindern, dass es eines Tages zu einem völligen Desinteresse am Germanistikstudium kommt. Es wird zwar argumentiert, man solle die Germanistik nicht mit einer fachsprachenorientierten Ausbildung beauftragen, aber welche Berufe werden dann später für Germanisten offen stehen, abgesehen vom Lehramt? Längst nicht jeder, der Germanistik studiert, wird Deutschlehrer an der Schule oder Wissenschaftler an der Hochschule. Darüber hinaus sind die Fachsprachen „Wirtschaftsdeutsch“ und „Deutsch für den Tourismus“ sowie das schon an einigen deutschen Universitäten etablierte Studienfach „Interkulturelle Kommunikation“ Mikrobereiche der Soziolinguistik, die eine Spezialisierung innerhalb der Linguistik darstellt und Überschneidungen mit anderen Kerndomänen aufweist. Die Ausbildung solcher Schlüsselqualifikationen als idealtypische Zielsetzungen steht selbstverständlich nicht im Widerspruch zum Germanistikfachwissen. Wir bewegen uns also immer noch im Bereich der Germanistik, jedoch mit einer besseren Berufsperspektive. In diesem Zusammenhang sieht Pleines die Aufgabe der Hochschulgermanistik eher darin, ,aus ihrer fachwissenschaftlichen Zuständigkeit heraus Grundlagen und Reflexionsdimensionen für solche eher anwendungsbezogenen Ausbildungsinstitutionen bereitzustellen und somit auch für den germanistisch qualifizierten Nachwuchs in diesen Sektoren die erforderlichen Qualifikationsmöglichkeiten zu schaffen“ (Pleines 1990:46, zitiert in Jai-Manssouri 2003:4748). 
Die „klassischen“ germanistischen Kurse, wie literatur- und sprachwissenschaftliche Lehrveranstaltungen, werden auf Grund ihres traditionell geisteswissenschaftlichen Charakters des Faches im Curriculum beibehalten. Diesen Kursen sollte jedoch weniger Gewicht beigemessen werden. Deshalb ist es erforderlich, die Curricula des Faches Deutsch in den marokkanischen Universitäten $\mathrm{zu}$ revidieren und $\mathrm{zu}$ aktualisieren. Eine Alternative wäre ein Studiengang, der die Fächer Wirtschaftsdeutsch, Deutsch für den Tourismus und interkulturelle Kommunikation kombiniert. Der neue Studiengang wird als ein praxis- und berufsorientierter Studiengang konzipiert, der aus sechs Modulen im Hauptstudium besteht, und lässt sich wie folgt darstellen:

Abb. 1. Lehrangebot im Studiengang Wirtschaftsdeutsch, Deutsch für den Tourismus und interkulturelle Kommunikation

\begin{tabular}{|c|c|c|c|}
\hline & Wirtschaftsdeutsch & Deutsch für den Tourismus & $\begin{array}{l}\text { Interkulturelle } \\
\text { Kommunikation }\end{array}$ \\
\hline & Basismodul & Basismodul & Basismodul \\
\hline Semester 5 & $\begin{array}{l}\text { - Firmenpräsentation } \\
\text { - Lesen von } \\
\text { Wirtschaftstexten } \\
\text { - Grafikkommentierung } \\
\text { - Handelskorrespondenz und } \\
\text { Vorstellungsgespräche } \\
\text { - Telefonkommunikation }\end{array}$ & $\begin{array}{l}\text { - Mündliche und schriftliche } \\
\text { Kommunikation in der } \\
\text { Tourismusbranche } \\
\text { - Geographie des Tourismus } \\
\text { - Tourismuspolitik }\end{array}$ & $\begin{array}{l}\text { - Interkulturelle Kommunikation: } \\
\text { Eine Einführung in Methoden und } \\
\text { Konzepte } \\
\text { - Interkulturelles Lernen im } \\
\text { Fremdsprachenunterricht } \\
\text { - Vergleichende Mentalitätsstudien } \\
\text { (Deutsche und Araber) }\end{array}$ \\
\hline \multirow[b]{2}{*}{ Semester 6} & Erweiterungsmodul & Erweiterungsmodul & Erweiterungsmodul \\
\hline & $\begin{array}{l}\text { - Auftragsabwicklung (von } \\
\text { Anfrage bis zur Zahlung) } \\
\text { - Führen von Mitarbeiter-, } \\
\text { Kunden- und } \\
\text { Verkaufsgesprächen } \\
\text { - Wirtschaftsleben in } \\
\text { Deutschland } \\
\text { - Professionelle Übersetzung } \\
\text { (Wirtschaft und Handel) } \\
\text { - Ein Praktikum und eine } \\
\text { praxisbezogene } \\
\text { Projektarbeit. }\end{array}$ & $\begin{array}{l}\text { - Betriebswirtschaft und } \\
\text { Rechnungswesen } \\
\text { - Tourismusmarketing } \\
\text { - Computer-Rechnungssysteme } \\
\text { - Professionelle Übersetzung } \\
\text { (Wirtschaft und Handel) } \\
\text { - Praktikum und eine } \\
\text { Praxisbezogene Arbeit }\end{array}$ & $\begin{array}{l}\text { - Interkulturelle } \\
\text { Wirtschaftskommunikation } \\
\text { - Interkulturelles Marketing } \\
\text { - Interkulturelle Nonverbale } \\
\text { Kommunikation } \\
\text { - Professionelle Übersetzung } \\
\text { (Wirtschaft und Handel) } \\
\text { - Praktikum in einem } \\
\text { internationalen } \\
\text { Arbeitskontext/Praxisbezogene } \\
\text { Projektarbeit }\end{array}$ \\
\hline
\end{tabular}

Das Ergänzungsfach „Wirtschaftsdeutsch, Deutsch für den Tourismus und interkulturelle Kommunikation" ist ein grundlegender interdisziplinär Studiengang. Er ergänzt das Kernstudium „Germanistik“ um eine anwendungsbezogene, an internationalen Berufszielen orientierte Komponente. Im Ergänzungsfach müssen jeweils ein Basismodul und ein Erweiterungsmodul in drei Studienbereichen des Faches absolviert werden. Der Studiengang ist konsekutiv aufgebaut. Das heißt: Die Lehrveranstaltungen innerhalb der Module bauen 
inhaltlich aufeinander auf. Basismodule sollen daher im fünften Semester und Erweiterungsmodule im sechsten (Abschlusssemester) studiert werden. Die Module ergänzen sich nicht zuletzt durch Konvergenz der Studieninhalte in idealer Weise, sondern sie sind auch inhaltlich miteinander verzahnt und führen zum Erwerb grundlegender Kenntnisse im Tourismusbereich sowie zur Befähigung zur erfolgreichen Gestaltung interkultureller Berufsfelder in der Wirtschaft.

Der Aufbau des Curriculums und die Struktur des Lehrplans sind jedoch noch konkreter und im Detail mit den Kollegen vor Ort zu besprechen. Vorausgeschickt seien hier Gedanken zum anzustrebenden Profil vom neuen Studiengang:

- Welche Einheiten könnten schon im vierten Semester eingeführt werden?

- Werden sich die Studierenden im letzten Semester je nach Interesse in ein oder zwei Ausbildungsbereiche trennen oder in einem gemeinsamen Bereich studieren?

- Welche neuen bzw. modernen Kurse sollen in die Lehrpläne eingeschoben werden, z.B. entsprechende EDV-Kurse?

Sollte dieser Studiengang zustande kommen, müssten die Vertreter der Germanistikabteilungen an einen Tisch gebracht werden, damit sie gemeinsam die Einrichtung eines neuen Germanistikprogramms in der Masterstufe (Aufbaumodule) diskutieren können. Das Aufbaustudium versteht sich als eine Fortsetzung eines berufsorientierten Studiengangs und ist zugleich der Ort, wo eine transdisziplinäre Zusammenarbeit zwischen verschiedenen Bereichen verstärkt möglich sein wird. Eine Zusammenarbeit zwischen den Fakultäten in Casablanca und Rabat ist in diesem Zusammenhang denkbar, denn die geographische Nähe und die gute Verkehrsverbindung ermöglichen dem Lehrpersonal die Durchführung dieses Projekts.

Auf diese Weise würde die Germanistik in Marokko so attraktiv wie möglich gestaltet, so dass sie der Nachfrage der Studierenden entspricht. Ansonsten muss die Germanistik ihre Daseinberechtigung in Frage stellen lassen, wenn das Fach ineffektiv arbeitet, vor allem wenn man die Tatsache berücksichtigt, dass die Universität finanzielle Mittel in einer Pauschalsumme für den jährlichen Haushalt erhält, der selbständig von der Universität festzusetzen ist. Voraussichtlich reichen die staatlichen Mittel nicht aus. Das bedeutet, die Universität kann künftig die Germanistik nicht finanzieren, wenn jedes Jahr ein stärkerer Rückgang der Einschreibungen für Germanistik zu verzeichnen ist.

Das Studienprogramm in Germanistik soll attraktiv, aktuell, sinnvoll und zeitökonomisch gestaltet werden, damit sich mehr Interessenten für Germanistik in Marokko finden lassen. Daraus könnte sich eine stärkere Motivation ergeben. Die neue Studienwahl und die damit verbundenen neuen Studieninhalte erzeugen bei den Studierenden positive Zukunftsaussichten und tragen dazu bei, sie stärker zu motivieren. Das Fachstudium, das auf Tätigkeiten in der Industrie, im Handel und im Tourismus vorbereitet, ermöglicht dem Studierenden, ,den Schwerpunkt auszusuchen, der ihn motiviert und von dem er begründet meint, dass er ihm für sein späteres Berufsleben nützt" (Jai-Mansouri 2003:48). Solche Berufsperspektiven eines Germanistikstudiums würden Deutsch in der Schule und Oberschule ebenfalls positiv beeinflussen. Die Motivation muss jedoch stärker unterstützt und entwickelt werden. Zu den motivationsfördernden Faktoren gehört in erster Linie die Ausrichtung an den gesellschaftlichen Bedürfnissen. Es ist Aufgabe des Lehrers, den Schülern Vorteile und Nutzen des Deutschen für verschiedene Berufe aufzuzeigen. Auf diese Weise kann sich eine 
Begeisterung beim Erlernen des Deutschen entwickeln, was sich positiv auf die Einstellung und Motivation der Schüler auswirken wird. Davon kann die Germanistik ebenso profitieren, weil die Anzahl der Interessenten an der Germanistik mit guten Deutschkenntnissen dann allmählich steigen wird.

\section{Schlussbemerkungen: Chance der deutschen Sprache in Marokko und Tendenz in der Zukunft}

Der Reformentwurf von 2000 hat die Nachteile des traditionellen Studiums erkannt und legt deshalb viel Wert auf eine Ausbildung mit Berufsperspektiven. Die gegenwärtige Reform hat einiges erreicht, z.B. wird „dem Universitätslehrkörper die Erstellung von Curricula, die Definition ihrer Inhalte und pädagogischen Elemente ebenso wie die Entscheidung über die passende Prüfungsform von erbrachten Leistungen überlassen“"(Jai-Mansouri 2003:48).

Paradoxerweise werden Zielsetzungen definiert, die der im Entwurf intendierten stärkeren Berücksichtigung des Praxis- und Anwendungsbezugs nicht entsprechen, obwohl die Vermittlung einer instrumentellen Basis für die Ausbildung eines späteren Berufs in der heutigen technologischen und globalisierten Welt den Studierenden zugute kommt. Man wird lediglich vom Zertifikatssystem zum Modulsystem mit denselben inhaltlichen Curricula übergehen. Verändert wurde lediglich der Rahmen dieser Curricula. Ein Ausweg aus dieser Kontroverse liegt m. E., abgesehen von den oben genannten Verbesserungsvorschlägen, in einer Veränderung der Einstellung der spezialisierten Lehrkräfte, damit eine Professionalisierung des Studienangebots realisiert werden kann. Die Verwirklichung dieses Schritts ist jedoch vor allem von der staatlichen Politik abhängig. Darüber hinaus sollten sich die Hochschullehrer im Rahmen der Angebote des DAAD (Deutscher Akademischer Austauschdienst) Gedanken über Weiterbildungsmöglichkeiten, z.B. in den Bereichen Wirtschaftsdeutsch, Handel, Deutsch für den Tourismus und interkulturelle Kommunikation, machen. Dies setzt jedoch voraus, dass man diese Ausbildung als Herausforderung und Bereicherung und nicht als Belastung empfindet.

Für den neuen Studiengang „Wirtschaftsdeutsch, Deutsch für den Tourismus und interkulturelle Kommunikation" ist vor allem in den Bereichen Forschung und Entwicklung, aber auch in Bezug auf Möglichkeiten der Praktikantenentsendung, eine enge Zusammenarbeit mit Institutionen und Unternehmen der Wirtschaft von besonders großer Bedeutung. Hier ist insbesondere daran zu denken, dass die staatlichen Mittel voraussichtlich nicht ausreichen, weshalb Einnahmen von anderen Quellen an Bedeutung gewinnen werden. Voraussetzung dafür ist, dass die Universität ihre Studiengänge so attraktiv wie möglich gestaltet, so dass sie dem Bedarf und der Nachfrage der Studierenden entsprechen. Eine traditionelle Auffassung von Germanistik oder den Geisteswissenschaften ist überholt und kann im Zeitalter der Globalisierung mit den Entwicklungen nicht mehr Schritt halten. Es handelt sich in Wirklichkeit um die Krise der vermittelnden Institution Universität und insbesondere der Geisteswissenschaften. Diese sind, was ihren inneren Aufbau und ihre Zielsetzungen betrifft, zum Teil noch eine Einrichtung der Nachkolonisationszeit als Ort der damals etablierten Nationalphilologien. Nicht aus dem Innern eines jeden Faches heraus ist eine Reform der Studienpläne möglich, sondern im ständigen fächerübergreifenden Dialog. Dadurch wird in Zukunft eine erhöhte Flexibilität gewährleistet, so dass neue Fächerkombinationen nach dem Modell Hauptfach/Nebenfach möglich sein werden und 
folglich die Lerner darauf vorbereitet werden, in einer globalisierten Informationsgesellschaft zu leben, die durch internationale Konkurrenz geprägt ist.

Auf deutscher Seite sollte man überlegen, ob die einseitige Orientierung nach Osten nicht dazu führt, dass man einen interessanten Zukunftsmarkt vernachlässigt, der eine Reihe von Vorteilen zu bieten hat, wie z.B. Nähe zu Europa, politische und wirtschaftliche Stabilität, eine leistungsfähige Arbeitnehmerschaft, funktionierende Infrastruktur usw. Eine der wichtigsten Aufgaben des DAAD als Mittler der Auswärtigen Kulturpolitik liegt darin, die deutsche Sprache zu fördern. Dementsprechend ist es wünschenswert, dass sich der DAAD in Zusammenarbeit mit dem Goethe-Institut für die Internationalisierung der Germanistik und der deutschen Sprache konkret einsetzt und so gemeinsam mit Marokko die Zukunft der deutschen Sprache in unserem Land sichern hilft. ${ }^{3}$

Sollten wenigstens diese Rahmenbedingungen geschaffen werden, können wir nicht nur Deutsch als Fremdsprache retten, sondern auch einen großen Beitrag zur Entwicklung des Germanistikstudiums in Marokko leisten. Dadurch würde man dem Land und seinen jungen Menschen einen Zugang zu einem der großen Wirtschaftsräume Westeuropas, einen Zugang zu Informationen und Kenntnissen verschaffen, die der Entwicklung des eigenen Landes dienen können.

\section{Anmerkungen}

1. Dies ist insbesondere in einem Käufermarkt wichtig, nach dem Motto: „If you just buy in Germany, you can speak Englisch. But if you want to sell in Germany, you must speak German“.

2. Wünschenswert wäre ein binationaler Studiengang „Interkulturelle Kommunikation Deutschland-Marokko“ nach dem Modell „Interkulturelle Kommunikation Deutschland-China“. Dieser deutsch-chinesische Masterstudiengang ermöglicht es Studierenden aus Deutschland und China, ihr germanistisches Masterstudium gemeinsam an Universitäten in beiden Ländern $\mathrm{zu}$ absolvieren. Nach einer obligatorischen Studienphase an der Georg-August-Universität Göttingen entscheiden sich die Studierenden für eine zweite Studienphase an der Universität Nanjing oder der Beijing Foreign Studies University. Das Studium schließt nach vier Semestern mit einem deutsch-chinesischen Double Degree ab. Der Studiengang wird von der Abteilung Interkulturelle Germanistik der Georg-August-Universität Göttingen in Zusammenarbeit mit der Deutsch-Abteilung der Universität Nanjing sowie der Beijing Foreign Studies University angeboten. Die Entwicklung wurde im Rahmen des EUProgramms Asia-Link von der Europäischen Kommission gefördert und vom Bundesministerium für Bildung und Forschung als Pilotprojekt in den Geisteswissenschaften unterstützt.

3. In Usbekistan z.B. fördert der DAAD durch fachspezifische Programme die Germanistik- und Deutschabteilungen an den Hochschulen. In diesem Zusammenhang führte das DAAD IC Taschkent im November 2006 ein fünftägiges Fortbildungsseminar zum Thema „Wirtschaftsdeutsch“ für Germanistiklehrende an usbekischen Hochschulen in der Universität für Diplomatie und Weltwirtschaft, Taschkent durch. Die Teilnehmer aus der gesamten Republik arbeiteten eine Woche sehr intensiv und wurden durch DAAD-Lektoren und führende deutsche Spezialisten (Siemens, Commerzbank, Deutsche Botschaft) mit aktuellen Fakten und sprachlichen 
Feinheiten vertraut gemacht. In Marokko sollte der DAAD auch solche Veranstaltungen mit ähnlichem Engagement wie in Usbekistan fördern.

\section{Bibliographie}

Pleines, J. 1990. Wozu Germanistik in Marokko? In J. Pleines (Hg.) Germanistik in Maghreb (DAAD-Dokumentationen und Materialien, 20). Bamberg: DAAD. pp. 41-55.

Jai-Mansouri, R. 2003. Das Germanistikstudium in Marokko mit Blick auf die geplante Universitätsreform. In DAAD (Hg.) Germanistiktreffen: Deutschland - Arabische Länder, Iran. Bonn: DAAD. pp. 37-50.

Wilsch, K. 2003. Aspekte einer realitätsnahen Ausbildung von Germanisten und Übersetzen in Marokko. In DAAD (Hg.) Germanistiktreffen: Deutschland - Arabische Länder, Iran. Bonn: DAAD. pp. 199-205.

Zhao, J. 2002. Wirtschaftsdeutsch als Fremdsprache. Ein didaktisches Modell. Tübingen: Narr.

\section{Biographical note}

Since December 2001 I am a lecturer in German and business German at the University of Casablanca. After my studies in anglistics and German language and literature I received my Ph.D. from the University of Heidelberg in 2000, based on my work "Forms of politeness in the interaction between Germans and Arabics". After further education in Freiburg I worked as a German teacher for business German in 2002 at the Goethe Institute. My current research focuses on sociolinguistics, language teaching and globalization. 